\title{
A Delphi Consensus Approach for Difficult-to- Treat Patients with Severe Hemophilia A without Inhibitors
}

\author{
Sreenivas $\mathbf{P}$ Veeranki \\ Priti Pednekar' \\ Marlon Graf' \\ Rifat Tuly' \\ Michael Recht $\mathbb{D}^{2,3}$ \\ Katharine Batt ${ }^{\prime}$
}

'PRECISIONheor, Los Angeles, CA, USA;

${ }^{2}$ American Thrombosis and Hemostasis

Network, Rochester, NY, USA; ${ }^{3}$ The

Hemophilia Center, Oregon Health \&

Science University, Portland, OR, USA
Correspondence: Priti Pednekar PRECISIONheor, III 00 Santa Monica Blvd, Ste 500, Los Angeles, CA, 90025 , USA

Tel +I 2674383588

Email Priti.Pednekar@precisionvh.com
Introduction: Over the past decade, there has been an increase in novel therapeutic options to treat hemophilia A. It is still unclear how these novel treatments are used in the management of patients with hemophilia A, particularly those with challenging clinical scenarios who are typically excluded in clinical trials.

Purpose: This study aimed to understand the areas of consensus and disagreement among hematologists regarding the preferences toward therapeutic approaches for difficult-to-treat patients with severe hemophilia A without inhibitors.

Patients and Methods: During February-June 2020, a three-round modified Delphi study was conducted to generate consensus among 13 US experts in the field of hemophilia. Experts were asked about their preferences toward therapeutic options for patients with challenging clinical situations, including age-related morbidities (eg, myocardial infarction, joint arthropathy), increasing demand for high-impact physical activities, early onset osteoporosis, and newborns with hemophilia A. Consensus was defined as $\geq 75 \%$ agreement between the panelists.

Results: Consensus was reached on many, but not all cases, leaving uncertainty about appropriateness of therapeutic approaches for some patients where clinical evidence is not available or driven by physicians' or patients' preferences toward therapeutic options. A majority of panelists preferred FVIII replacement therapy rather than emicizumab prophylaxis for the challenging cases presented due to established evidence on safety, efficacy, and level of bleed protection for FVIII treatment.

Conclusion: Recommendations emerging from this study may help guide practicing hematologists in the management of challenging hemophilia A cases. Future studies are needed to address treatment options in the clinical cases where no consensus was reached.

Keywords: emicizumab, expert elicitation, FVIII, treatment, management

\section{Introduction}

Patients with hemophilia A (PwHA) experience spontaneous bleeds resulting in a significant clinical burden and poor quality of life (QoL). ${ }^{1,2}$ While Factor VIII (FVIII) replacement treatment, the standard of care for the management of hemophilia $\mathrm{A}^{3}$ is safe and effective, it carries a known risk of inhibitor development (approximately $30 \%$ of PwHA develop inhibitors), profoundly impacting the patient's QoL, morbidity and mortality with increased need for venous access and infusions, despite less effective bleed control. ${ }^{4,5}$ These challenges have led to the emergence of novel, non-factor replacement therapies that can impact bleeding at 
different stages of the coagulation cascade, often times with an easier method of delivery or decreased frequency of administrations. ${ }^{3,4}$

The increased availability of treatment options for PwHA presents a unique opportunity to create individualized, patient-centered regimens. However, the accelerated pace of innovation also demonstrates physician awareness of novel treatment options along with combination with existing treatments may be lacking, particularly when considering the management of severe PwHA (SPwHA) without inhibitors. In routine clinical practice, therapeutic decisions are often challenged by real-world clinical needs, particularly in PwHA without inhibitors with more severe bleeding phenotype, who are involved in high-impact physical activities, and who report arthropathy symptoms or have age-related comorbidities such as cardiovascular diseases, osteoporosis, renal failure, etc. Uncertainty in management of such cases in the absence of robust guidelines, leads to a high degree of variability in therapeutic approaches and poor outcomes.

The current study employed a Delphi method to address uncertainties in the treatment and management of high-risk cases of SPwHA without inhibitors. The Delphi method is recommended for use in obtaining consensus in the healthcare setting when "gold-standard" evidence is not available or available evidence lacks sufficient details to apply to the subset of patients observed in clinical practice. $^{6,7}$ The study also identified patients' and clinical characteristics that influence physicians' treatment decision-making in the management of PwHA.

\section{Study Participants and Methods Delphi Panel Participants Selection}

The Delphi panel participants were recruited through recommendations from two hemophilia experts and based on the existing literature. This study included expert panel members who a) were board-certified physicians in either adult or pediatric hematology b) had current or previous clinical practice for at least 5 years, c) had at least $50 \%$ of time or effort dedicated to treating patients, d) treated at least 15 patients with hemophilia during the past 12 months, e) had at least 10 relevant peer-reviewed publications in the evaluation and treatment management of patients with hemophilia, and $\mathrm{f}$ ) practiced in the United States only. Experts who were unable to speak or read English were not included in the panel. A total of 51 potential panelists were identified and contacted with an introductory email outlining the study and participation requirements.

\section{Delphi Panel Instrument}

A targeted literature review was conducted to identify currently available treatments and their advantages and limitations, guidelines for the management of hemophilia A, factors influencing decision-making of hematologists while selecting or switching treatments, and challenging clinical scenarios in SPwHA without inhibitors. Subsequently, a survey instrument was developed based on findings from the targeted literature review and inputs from two hemophilia experts with emphasis on challenging clinical scenarios or situations where treatmentdecision making has not been well established.

\section{Delphi Panel Implementation}

A modified Delphi panel consisting of three rounds of a web-based questionnaire was conducted between February and June 2020 (Figure 1). The Delphi survey was implemented using the web-based Delphi platformWelphi. ${ }^{8}$ All responses of panelists were kept anonymous and consent from panelists was obtained during each round. Experts who did not complete the Delphi survey round within the allotted time were not eligible to participate in the subsequent rounds. Panel members who agreed to participate were provided honoraria for participation in each round. The study was conducted in a double-blind manner, where the panel members had no knowledge about the study sponsor, and the study sponsor, as well, was not aware of the experts' names and their work- place organizations. Additionally, the study panelists were also blinded from their fellow panelists, thus all responses were anonymous.

Round 1 of the Delphi exercise was intended to establish the experts' baseline - a) preferences for different treatment options in challenging clinical scenarios involving SPwHA without inhibitors, b) assessment of outcomes associated with switching from factor replacement therapy to a nonfactor replacement option, and c) identification of key factors considered in the treatment decision-making process. Experts were asked to review each clinical scenario and provide their likelihood estimates for each therapeutic option, as per their clinical knowledge. Likelihood estimates ranged from 1-100 with lower values indicating an expert's lower preference for a particular treatment option in his/her patients. For a few survey questions, experts were asked to rank factors affecting treatment decision-making in 

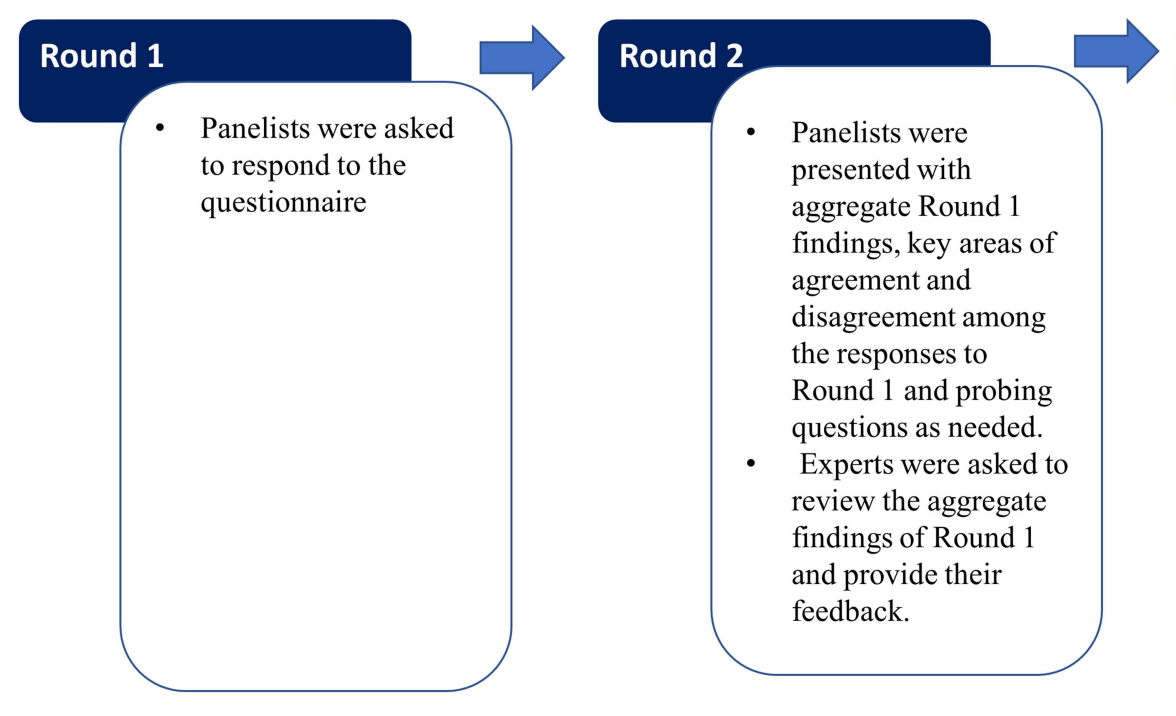

Round 3

- Panelists were asked to reconsider round 1 responses based on Round 2 feedback

- Panelists were asked to submit final responses to same questionnaire as in Round 1

- Panelists were asked to complete demographic and professional background survey

Figure I Modified Delphi process.

hemophilia A. In round 2, an online forum discussion was conducted in which experts were presented with aggregate group responses from round 1 as well as additional probing questions where needed. Experts were asked to review the aggregate findings from round 1 and provide feedback on any response(s) they chose, using the "comments" feature in Welphi. This round facilitated discussion among the panelists, allowing them to argue for different treatment approaches in any of the clinical scenarios, while also sharing their individual clinical experiences and expertise while avoiding oversaturation and participation fatigue. ${ }^{9,10}$ In round 3, the experts completed the same questionnaire as that in round 1, but now responding with the additional knowledge and discussion gleaned from round 2. Final responses were analyzed as described for round 1. Panel members were also asked to complete a brief demographic and professional background survey. Using the Department of Health and Human Services regulations, 45 CFR 46.104 (d)(2), Advarra Institutional Review Board (IRB) exempted this study from IRB oversight.

Descriptive analyses were conducted to summarize panel members' characteristics, including demographics and expertise in hemophilia management. For clinical scenarios, panelists were asked to indicate appropriateness of treatment described. A scale from 1 to 100 was provided, where 1-50 ratings were considered "not appropriate or not recommended" and 51-100 ratings were considered as "appropriate/recommended". In analyzing the responses, consensus was considered to be present if at least $75 \%$ of panelists reported "not appropriate" or "appropriate" ratings during round $3 .{ }^{11-14}$ For likelihood questions, the median and interquartile range
(IQR) were also calculated. ${ }^{15}$ For ranking questions, variations in proportion of panelists choosing different treatment options or responses as high rank compared to lower ranks were analyzed to gauge the presence or absence of consensus among panel members. All analyses were conducted in the programs built into Welphi and Microsoft Office Excel.

\section{Results \\ Participant Characteristics}

Out of 51 hemophilia experts invited, 14 experts agreed to participate; 13 experts completed all three rounds of the Delphi study and therefore, formed a panel. Approximately 25 invited experts expressed interest in the study but could not participate due to clinical responsibilities encountered during the COVID-19 pandemic. All 13 panelists provided consent to participate in the study. A majority of the panelists were male $(69.2 \%)$, white (76.9\%) and aged 31-50 years (46.2\%) (Table 1). Most panelists $(53.8 \%)$ had been treating PwHA for 10-19 years. Most panelists (76.9\%) spent their time providing direct patient care in a hemophilia treatment center. Most panelists $(61.5 \%)$ treated both adult and pediatric PwHA and $46.1 \%$ panelists treated more than 50\% SPwHA without inhibitors during the past three months.

\section{Panelists' Preferences for Hemophilia Management in Challenging Scenarios PwHA with Cardiovascular Comorbidities}

The panel was asked to indicate their preferred therapeutic option for a 69-year-old SPwHA without inhibitors 
Table I Characteristics of Delphi Participants, Their Professional Background, and Hemophilia Patients They Treat

\begin{tabular}{|c|c|c|}
\hline Characteristic & $\mathbf{n}$ & $\%$ \\
\hline Total & 13 & \\
\hline \multicolumn{3}{|l|}{ Demographic characteristics } \\
\hline \multicolumn{3}{|l|}{ Age group } \\
\hline $31-50$ years & 6 & $46.2 \%$ \\
\hline $51-70$ years & 6 & $46.2 \%$ \\
\hline $71-90$ years & 1 & $7.7 \%$ \\
\hline \multicolumn{3}{|l|}{ Sex } \\
\hline Female & 3 & $23.1 \%$ \\
\hline Male & 9 & $69.2 \%$ \\
\hline Other & 1 & $7.7 \%$ \\
\hline \multicolumn{3}{|l|}{ Race } \\
\hline White & 10 & $76.9 \%$ \\
\hline Asian & 2 & $15.4 \%$ \\
\hline Other & 1 & $7.7 \%$ \\
\hline \multicolumn{3}{|l|}{ Professional background } \\
\hline \multicolumn{3}{|l|}{ Year when medical license was obtained } \\
\hline $1950-1990$ & 4 & $30.8 \%$ \\
\hline $199 \mid-2000$ & 4 & $30.8 \%$ \\
\hline Later than 2000 & 5 & $38.4 \%$ \\
\hline \multicolumn{3}{|c|}{ Number of years working in the field of hemophilia $A$} \\
\hline $10-19$ years & 7 & $53.8 \%$ \\
\hline 20-29 years & 5 & $38.5 \%$ \\
\hline$\geq 30$ years & 1 & $7.7 \%$ \\
\hline \multicolumn{3}{|l|}{ Principal practice location ${ }^{a}$} \\
\hline Hemophilia treatment center & 10 & $76.9 \%$ \\
\hline University hospital or university affliated clinic & 3 & $23.1 \%$ \\
\hline \multicolumn{3}{|c|}{$\begin{array}{l}\text { Number of hemophilia A patients treated at the principal practice } \\
\text { location }\end{array}$} \\
\hline$\leq 125$ & 5 & $38.5 \%$ \\
\hline $126-150$ & 2 & $15.4 \%$ \\
\hline $151-175$ & 1 & $7.7 \%$ \\
\hline$>175$ & 5 & $38.5 \%$ \\
\hline \multicolumn{3}{|c|}{$\begin{array}{l}\text { Characteristics of hemophilia patients treated at clinical practice } \\
\text { location }\end{array}$} \\
\hline \multicolumn{3}{|c|}{ Hemophilia A patient population typically treated } \\
\hline Pediatric & 5 & $38.5 \%$ \\
\hline Both pediatric and adult & 8 & $61.5 \%$ \\
\hline \multicolumn{3}{|l|}{ SPwHA managed during past three months } \\
\hline$\leq 40 \%$ & 4 & $30.8 \%$ \\
\hline $4 \mid-50 \%$ & 3 & $23.1 \%$ \\
\hline$>50 \%$ & 6 & $46.1 \%$ \\
\hline
\end{tabular}

(Continued)
Table I (Continued).

\begin{tabular}{|l|c|c|}
\hline Characteristic & n & $\%$ \\
\hline SPwHA prescribed emicizumab & 3 & $23.1 \%$ \\
\hline $6-10 \%$ & 5 & $38.4 \%$ \\
$11-20 \%$ & 1 & $7.7 \%$ \\
$21-30 \%$ & 4 & $30.8 \%$ \\
More than 30\%
\end{tabular}

Note: ${ }^{2}$ Principal practice location is the practice location where hematologist spends the most hours per week.

Abbreviation: SPwHA, severe patient with hemophilia A.

diagnosed with a non-ST-elevation myocardial infarction (current therapy: dual antiplatelet therapy (DAPT) with FVIII prophylaxis) (Table 2). The panel reached consensus around "FVIII prophylaxis to achieve desired FVIII levels and then episodic FVIII therapy along with DAPT" as not an appropriate therapeutic option for this patient $(84.6 \%$ panelists reported likelihood $\leq 50 \%$ ). During round 2 discussion, panelists argued that due to high risk of bleeding while on DAPT, continuous FVIII prophylaxis would be an appropriate therapy.

For a patient with recently documented $\mathrm{AF}$ with $\mathrm{CHA}_{2}$ $\mathrm{DS}_{2}$ VASc score $=2$, on episodic FVIII replacement therapy prior to the diagnosis of AF, panelists reached consensus that "continuing episodic FVIII replacement therapy only with DOACs" was not a recommended treatment (100.0\% panelists reported likelihood $\leq 50 \%$ ) (Table 2). The panel did not reach consensus on whether "emicizumab prophylaxis along with episodic FVIII and DOAC" was an appropriate treatment and reported in round 2 that emicizumab might provide more favorable coverage than FVIII prophylaxis in SPwHA with cardiovascular comorbidities; however, the risk of emicizumab-associated thrombotic complications in these patients is still unknown. During round 2, panelists expressed needing more information on pharmacodynamics and efficacy profile of emicizumab to reach consensus.

\section{PwHA with Increasing Demand for Physical Activity and Musculoskeletal-Related Disorders}

The panelists were asked which therapy they would prescribe to a 19-year old SPwHA without inhibitors with a history of joint bleeds yet interested in participating in high-intensity sport activities (current therapy: extended half-life FVIII prophylaxis) (Table 2). All panelists agreed that "FVIII prophylaxis with on-demand FVIII treatment post-bleeds" was not an appropriate treatment for this 


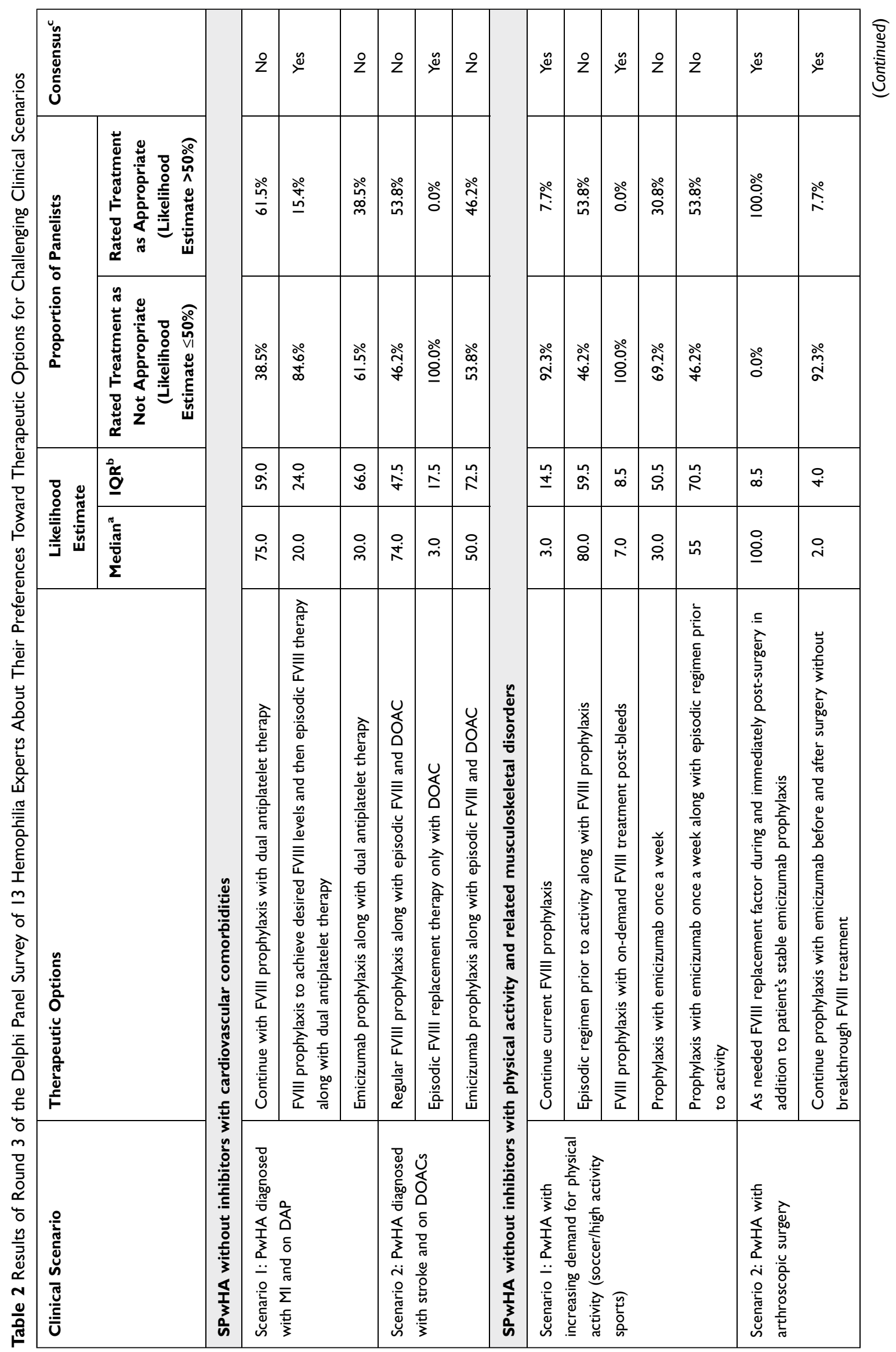




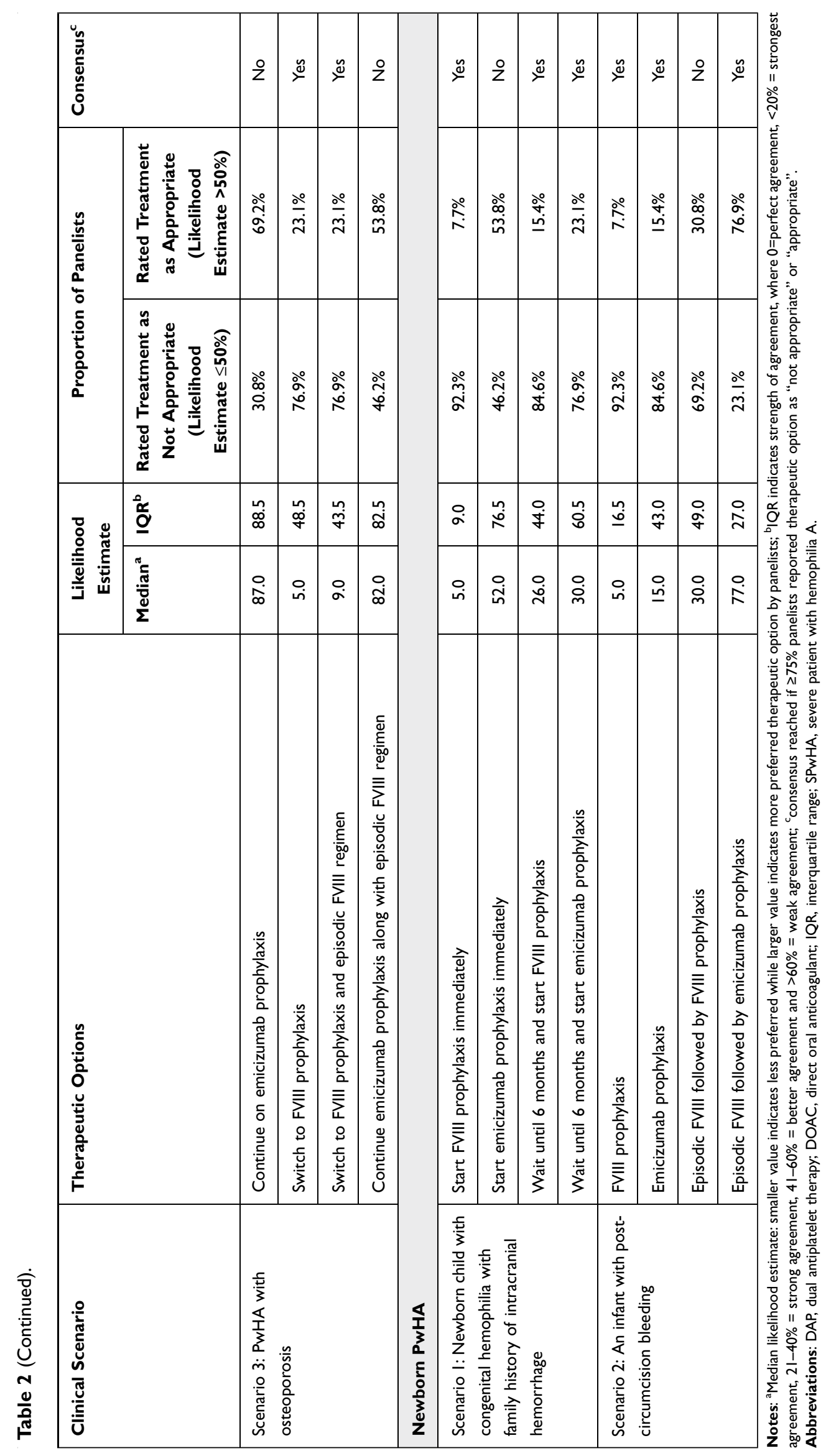


patient and during round 2, highlighted the need for preactivity FVIII bolus for these patients. During round 2, panelists discussed that emicizumab prophylaxis may protect patient from joint bleeds; however, in round 3, they did not reach consensus given implicit concerns with the cost of combined treatment as well as broad access/reimbursement issues.

The panel was asked about how they managed SPwHA without inhibitors scheduled for a joint surgery (current therapy: emicizumab prophylaxis). Based on available evidence and extensive clinical experience using FVIII peri-operatively, the panel reached consensus on the most appropriate treatment as "FVIII replacement therapy as needed during and immediately post-surgery in addition to patient's current, stable emicizumab prophylaxis" (100.0\% panelists reported likelihood $>50 \%$ ) (Table 2).

For SPwHA suffering early onset osteoporosis and treated historically with on-demand FVIII therapy but recently started on emicizumab prophylaxis, panelists reached consensus on "switching to FVIII prophylaxis" (76.9\% panelists reported likelihood $\leq 50 \%$ ) and "switching to FVIII prophylaxis along with episodic FVIII regimen" (76.9\% panelists reported likelihood $\leq 50 \%)$ as inappropriate treatment (Table 2). The majority of panelists $(69.2 \%)$ preferred continuing emicizumab prophylaxis with the hope that the patient's physical activity would increase and result in some bone remodeling, however consensus was not reached.

\section{Newborn PwHA}

For a newborn PwHA with a brother diagnosed with severe hemophilia, on FVIII prophylaxis with a prior history of intracranial hemorrhage, panelists reached consensus on a least recommended treatment as "starting FVIII prophylaxis immediately" (92.3\% panelists reported likelihood $<50 \%$ ) and "waiting until 6 months and starting FVIII prophylaxis" (84.6\% panelists reported likelihood $\leq 50 \%$ ) (Table 2). Panelists did not reach consensus on starting emicizumab prophylaxis immediately, however, the panel reached consensus on "wait until 6 months and start emicizumab prophylaxis" as not an appropriate therapeutic option (76.9\% panelists reported likelihood $\leq 50 \%$ ).

The panel, when asked how to manage a newborn PwHA with post-circumcision bleeding, reached consensus on "FVIII prophylaxis" (92.3\% panelists reported likelihood $\leq 50 \%)$ and "emicizumab prophylaxis" (84.6\% panelists reported likelihood $\leq 50$ ) as not recommended treatments (Table 2). A majority of panelists agreed that they would wait for the infant to be 6-9 months before starting FVIII prophylaxis. The panel reached consensus on "episodic FVIII prophylaxis followed by emicizumab prophylaxis" as a recommended treatment $(76.9 \%$ panelists reported likelihood $>50 \%$ ) with episodic FVIII bolus and provide prophylaxis through easy subcutaneous administration of emicizumab.

In round 2, panelists agreed that FVIII prophylaxis is predictable, has long-standing safety data and can be adjusted, however, panelists expressed their low preference toward FVIII prophylaxis due to the difficulty in accessing veins in infants and the risk of inhibitor development. During round 2 discussion, panelists reported that emicizumab prophylaxis is easy to administer subcutaneously in infants; however, there is a lack of clinical evidence around emicizumab's ability to protect infants from bleeding events and to prevent intracranial hemorrhage. At present, panelists noted that treatment decisionmaking in the management of newborn PwHA is largely based on the preferences of physicians and patients.

\section{Panelists' Opinions on Outcomes Related to Treatment Switching}

Based on currently available efficacy and safety data for emicizumab, the panel agreed to switch treatment from a FVIII therapy to emicizumab prophylaxis if a SPwHA without inhibitors had 4-5 bleeds during the previous year or had more than 5 bleeds during the previous year (Table 3). Panelists, however, did not reach consensus that SPwHA without inhibitors should be switched from FVIII therapy to emicizumab prophylaxis if a patient had $0-1$ bleeds or $2-3$ bleeds during the previous year. There is no standard cut-off for the number of bleeds during the previous year to determine when to switch from the FVIII treatment to emicizumab prophylaxis. Yet, panelists seemed to prefer emicizumab prophylaxis to provide protection against bleeds if patient had at least 4 bleeds during the previous year while on FVIII therapy. More data on efficacy and safety of emicizumab prophylaxis compared to FVIII treatment may further help clinicians to make treatment decisions. Panelists also reached consensus that switching from prophylaxis with factor replacement therapy to emicizumab is only likely to reduce bleeding events by $<10 \%$ or $10-40 \%$. Panelists did not reach consensus on whether switching prophylaxis from FVIII replacement therapy to emicizumab would impact medication adherence among SPwHA without inhibitors. 


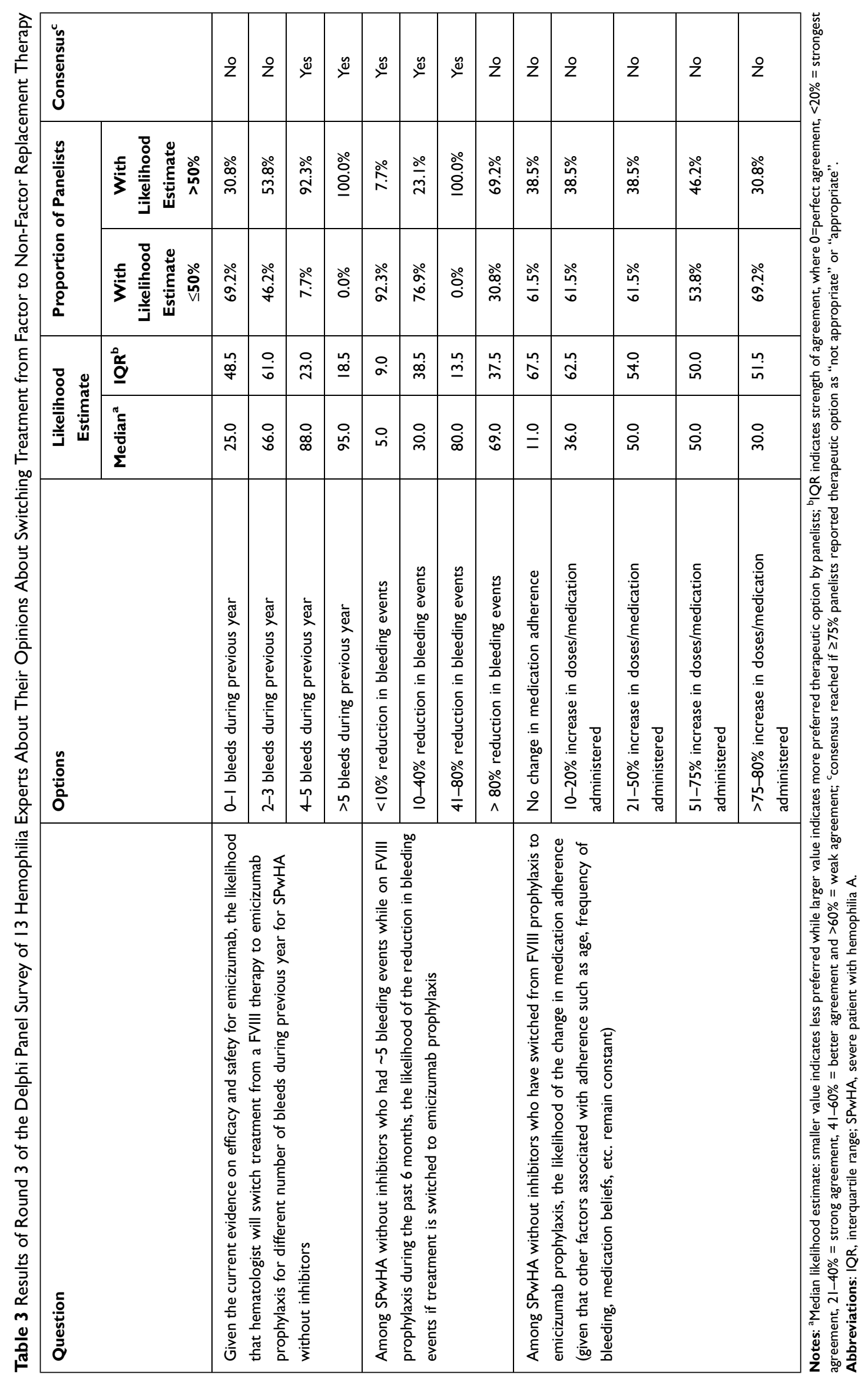




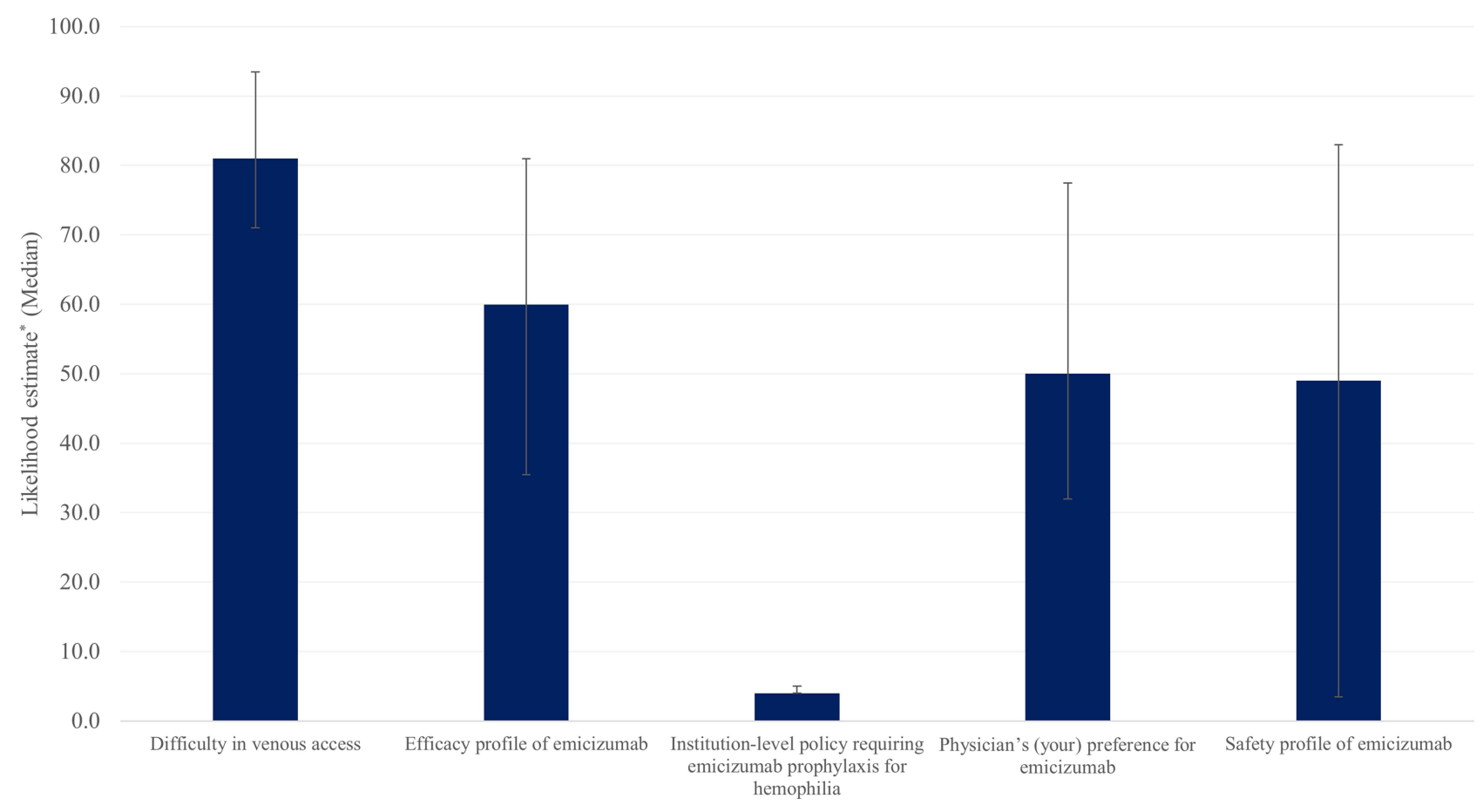

Figure 2 Factors considered by hematologists while switching SPwHA without inhibitors from FVIII prophylaxis to emicizumab prophylaxis. Note: "Panelists provided their likelihood estimates ranging from $\mathrm{I}-100$, where I means highly unlikely and 100 means extremely likely.

\section{Factors Affecting Treatment Decision-Making in SPwHA}

Panelists reached consensus that the primary factors considered when switching treatment from factor replacement therapy to non-factor treatment in SPwHA were difficulty in venous access (Figure 2), long-term efficacy and/or effectiveness, and physician or patient preferences toward treatment options (Figure 3). Regarding health system related factors, the majority of panelists (38\%) reported patients' knowledge of and preferences toward treatment options is most important to consider while switching treatment for SPwHA without inhibitors (Figure 4). While only $8 \%$ of panelists ranked institution-level policies (such as those related to prescribing procedures, dose distribution systems, outpatient prescription availability, etc.) as the most important health system related factors considered in treatment decision-making in management of SPwHA, approximately $23 \%$ panelists considered insurance coverage policies (such as those related to type of plan, access to and reimbursement of hemophilia treatments, out-of-pocket payment, management tactics employed in pharmacy or medical benefits by health plan) as major factors in treatment decisionmaking for SPwHA.

Results of round 1 are presented in Tables 4 and 5 .

\section{Discussion}

Existing guidelines for the management of hemophilia $A^{3,16}$ and clinical trials evaluating efficacy and safety of therapeutic options often do not provide recommendations on how to manage specific clinical scenarios. In particular, age-related comorbidities such as cardiovascular disease, early onset osteoporosis, increased bleeding risks associated with physical activity or a major surgery, or newborn infants with severe bleeding are often exclusion criteria for enrollment in randomized clinical trials. ${ }^{17}$ This further limits the applicability of evidence-based guidelines, possibly leading to misconceptions about available treatments. These gaps can only be bridged by achieving consensus among clinicians, typically drawn from their practice-based experience(s). Delphi techniques represent the most reliable consensus methods in healthcare. ${ }^{14,18-20}$ In the present Delphi study, cases of SPwHA without inhibitors of uncertain management were presented to a panel of 13 specialized clinicians in hemophilia, who answered a questionnaire on the appropriateness of treatment strategies. This is the first study of its kind, aimed at obtaining a consensus on complex and real-life cases of SPwHA without inhibitors, where definitive guidelines are not applicable. 
Immunogenicity (inhibitors or anti-drug-antibodies development to current therapy)
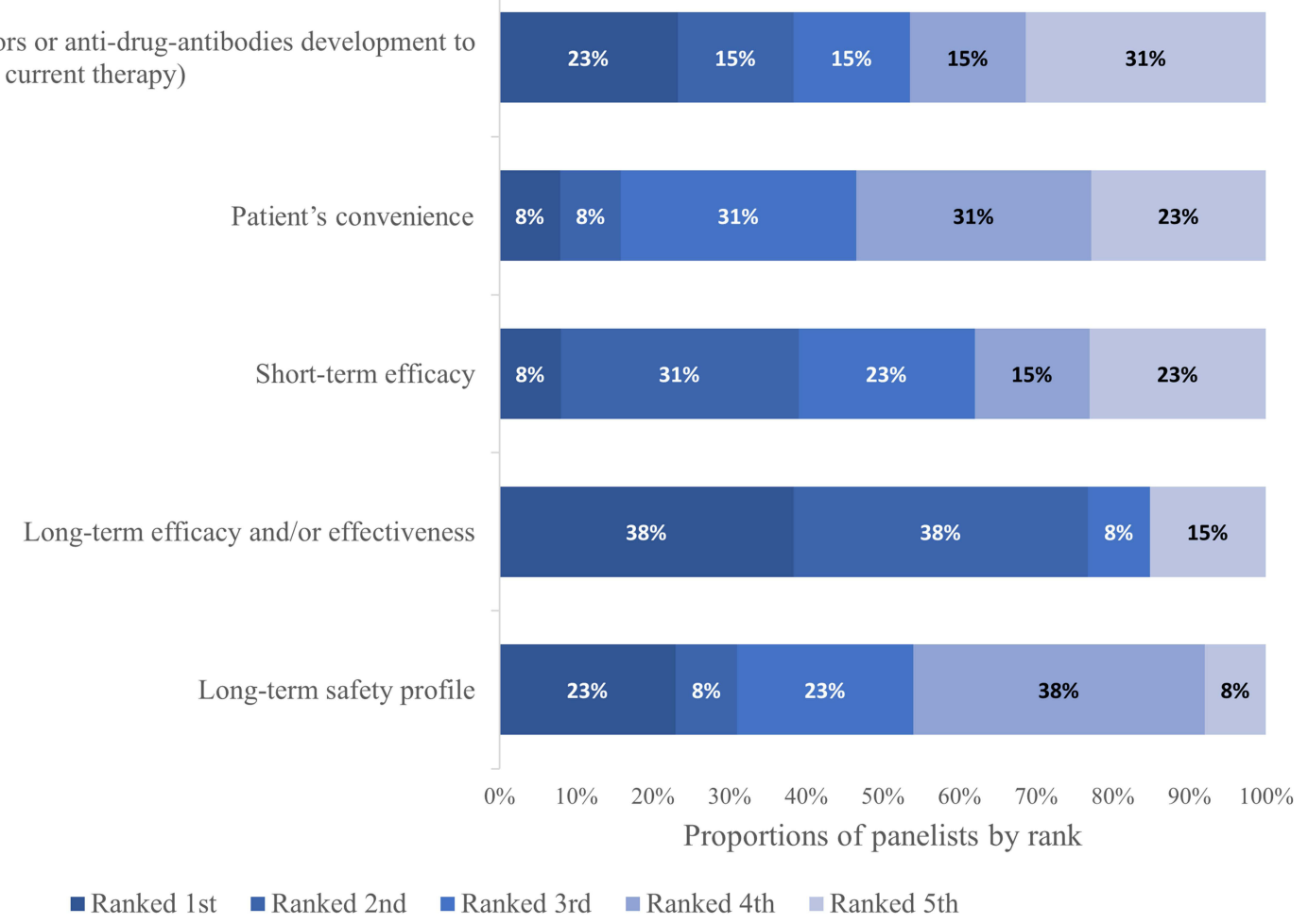

Figure 3 Hemophilia treatment characteristics considered by hematologists while switching treatment for SPwHA without inhibitors.

Pharmacists' preferences for specific treatment option over others

Organizational (hospital) policy regarding choice of specific treatment

Physician's (your) philosophy or inclination for specific treatment over others

Patients' knowledge of and preferences towards treatment options

Insurance coverage policies for specific treatments over others

- Ranked 1st Ranked 2nd
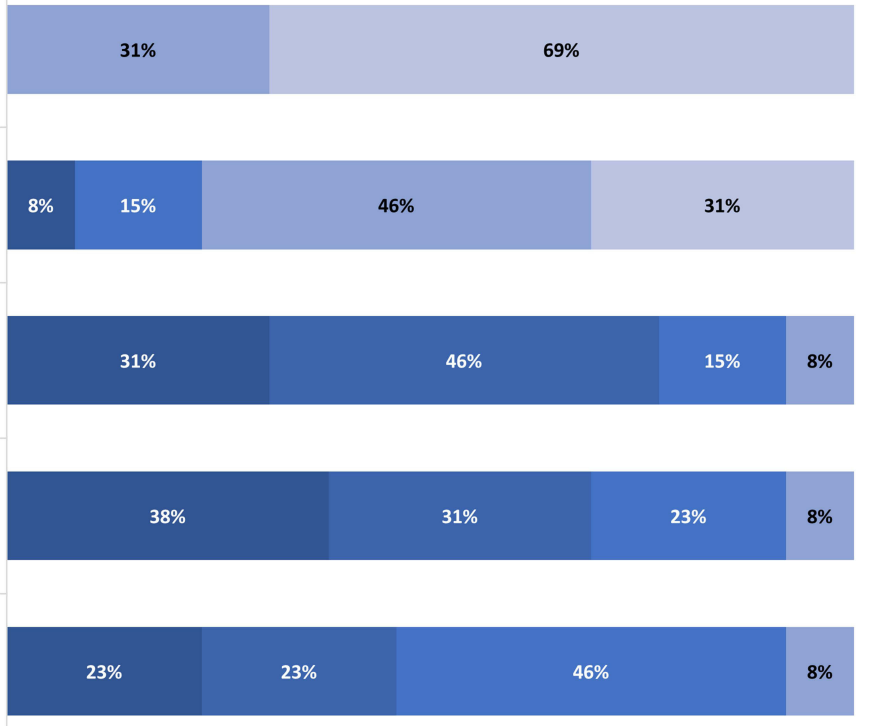

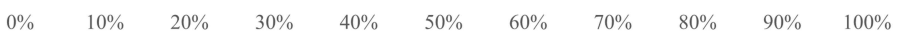

- Ranked 3rd $\quad$ Ranked 4th $\quad$ Ranked 5th

Figure 4 Health system related factors considered by hematologists while switching treatment for SPwHA without inhibitors.

For SPwHA with age-related morbidity such as cardiovascular disease (CVD), panelists reached consensus that episodic FVIII prophylaxis would not be adequate to reach targeted trough levels of FVIII required to avoid risk of bleeding associated with antithrombotic treatments. While the majority of panelists preferred FVIII prophylaxis, as 


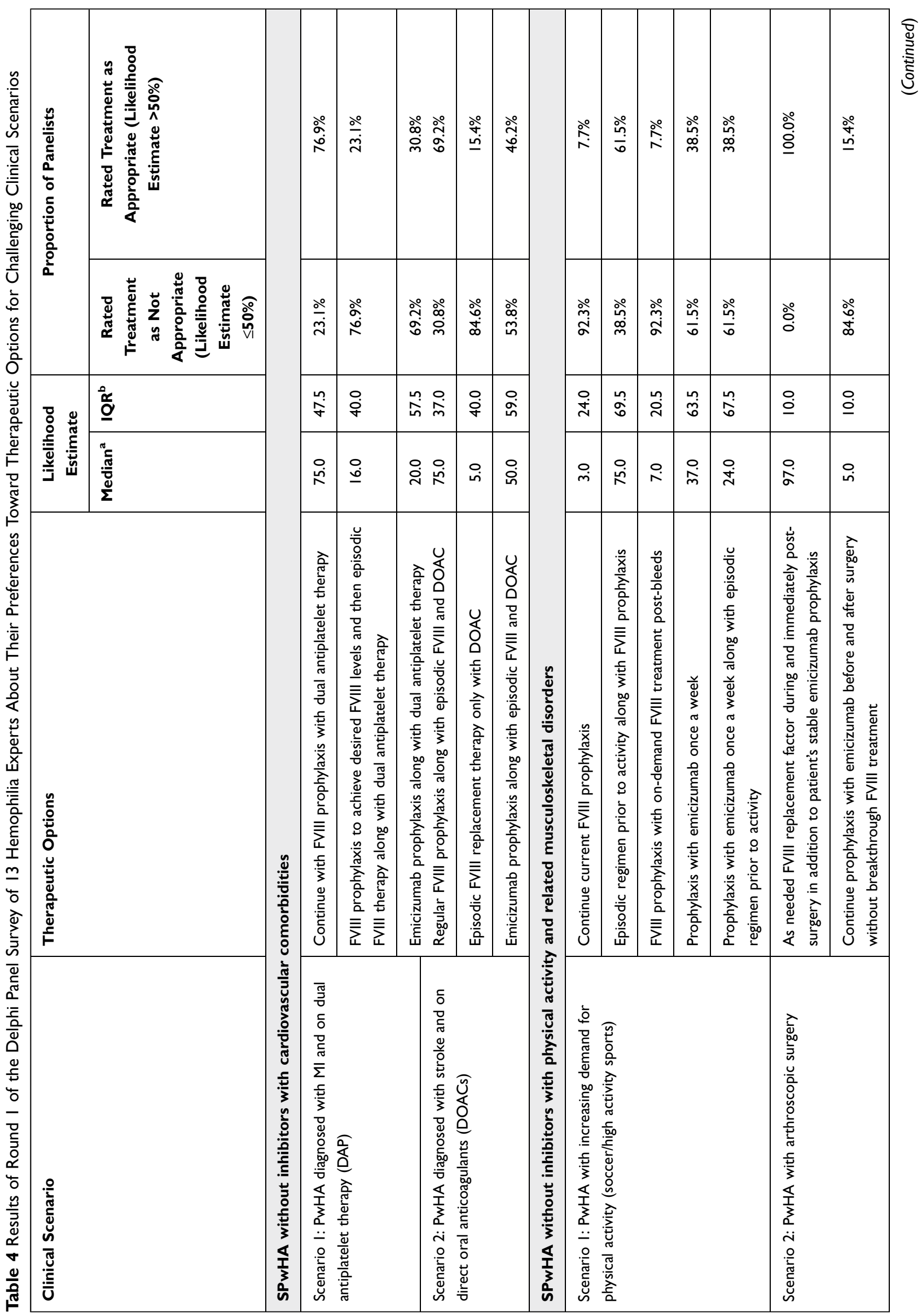




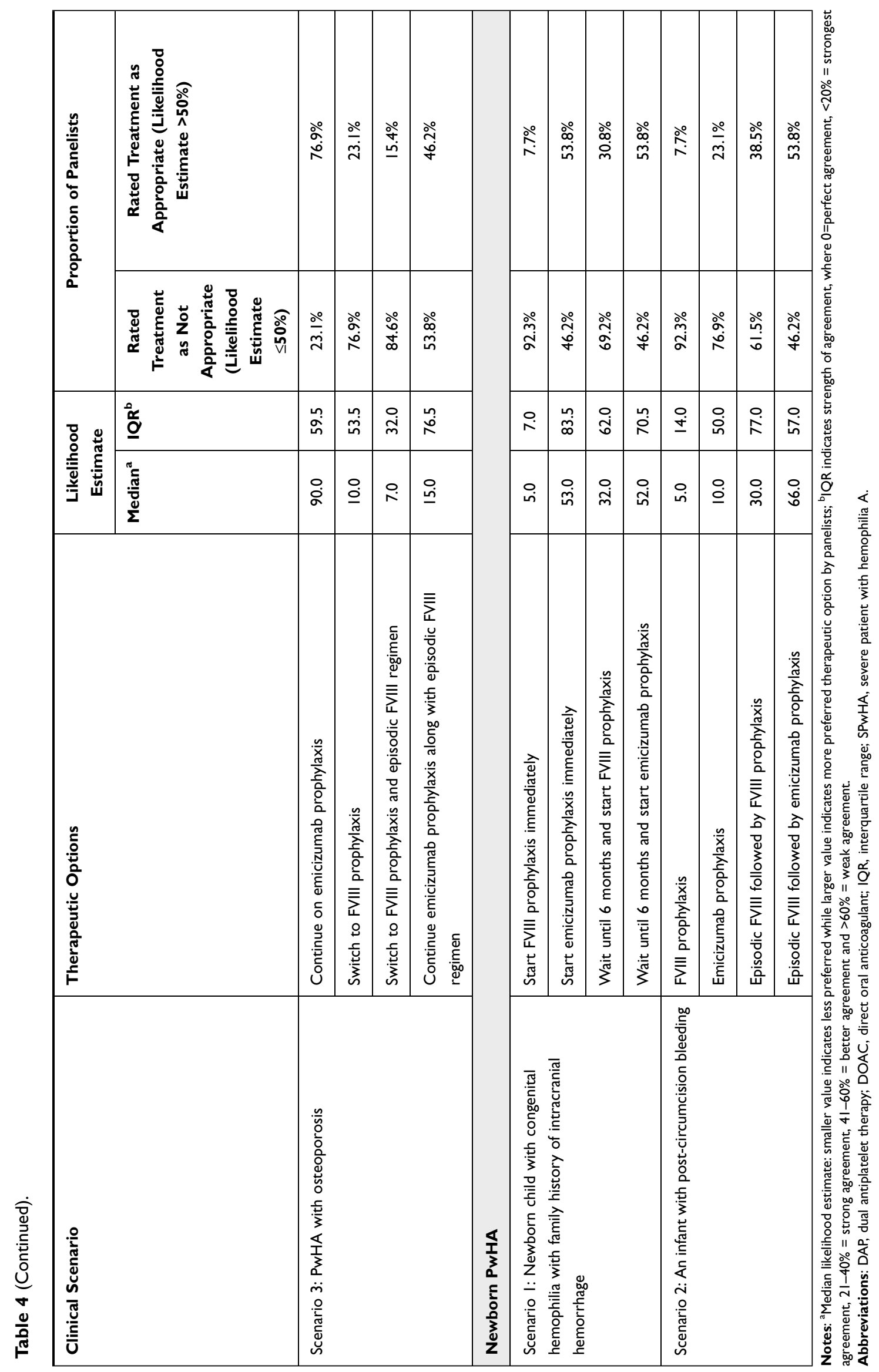




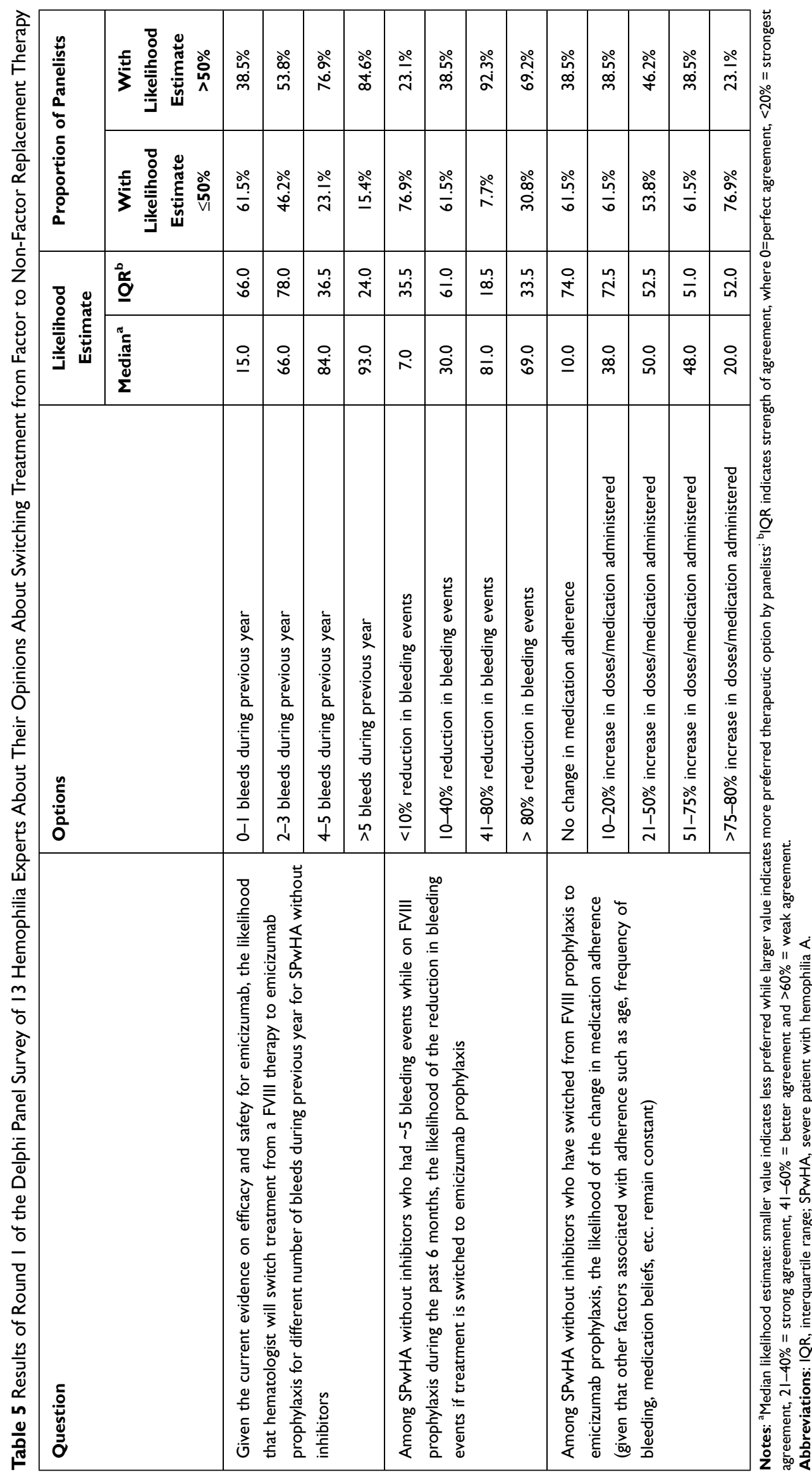


recommended by the World Federation of Hemophilia (WFH) guidelines ${ }^{21}$ and available limited evidence in the literature, ${ }^{22,23}$ a few panelists differed in their opinions around prophylaxis and consensus was not achieved. The uncertainty expressed by panelists toward emicizumab prophylaxis along with antithrombotic therapies was based on limited experience and lack of evidence-based guidelines on the use of emicizumab in elderly patients with CVD. Data on real-world adverse effects of emicizumab are still being collected; to this end, the thrombotic risk of emicizumab in elderly patients receiving antithrombotic treatments, remains unknown. ${ }^{24,25}$

In children and young adolescents with severe hemophilia A, physical activity, particularly high contact sports or prolonged activity, significantly increases the risk of bleeding. ${ }^{26}$ The management of hemophilia A becomes particularly challenging if a patient is not highly adherent to prophylaxis. ${ }^{27}$ For such patients, the panelists highlighted that this patient requires pre-activity episodic FVIII replacement therapy to mitigate the risk of bleeding; an on-demand, episodic regimen would not be adequate. Panelists needed more efficacy and/or effectiveness data to use emicizumab prophylaxis in these patients. HAVEN-3 trial results showed that emicizumab prophylaxis reduced joint bleeds as compared to no prophylaxis; however, no results were reported for young adults undergoing extensive physical activity. ${ }^{28}$

The surgical setting represents a challenge in the management of SPwHA due to the risk of peri-operative bleeding. The panel agreed that for a SPwHA without inhibitors undergoing a major surgical procedure such as arthroscopic surgery, FVIII replacement therapy is required during and post-surgery to secure hemostasis and wound healing; in such a scenario, emicizumab prophylaxis is not adequate as the hemostatic efficacy is not yet well established. Although HAVEN-3's intraindividual comparison of PwHA demonstrated emicizumab prophylaxis had significantly lower bleeds than previous FVIII prophylaxis, this cannot be extrapolated to a surgical setting, particularly given that the trial excluded patients experiencing bleeds due to surgery/procedures. ${ }^{28,29}$

SPwHA are also at increased risk of developing reduced bone mineral density, which poses greater risk of fractures and osteoporosis. ${ }^{30,31}$ For these patients, panelists reached consensus that switching to FVIII prophylaxis with or without episodic FVIII regimen would not be appropriate. While the role of FVIII in overall bone health is promising, there is very limited evidence supporting the role of FVIII prophylaxis in maintaining bone health. ${ }^{32}$ Although panelists preferred emicizumab prophylaxis with or without episodic FVIII more than FVIII prophylaxis with or without episodic FVIII, consensus was not reached. While a recent analysis of data from HAVEN-3 trial found that emicizumab promoted joint health in people with hemophilia $\mathrm{A},{ }^{28}$ the long-term effect of emicizumab prophylaxis on bone density compared to routine FVIII prophylaxis is still unknown.

\section{Strengths and Limitations}

Our study has several strengths. Our study panel consisted of experts in the field of hemophilia who have wide experience treating PwHA in real-world settings. Use of the Delphi method as a structured expert elicitation technique enabled capturing of perspectives and opinions across a broad spectrum of experts. This methodology facilitated an in-depth exploration of attitudes and opinions that is not possible in quantitative surveys. Anonymity among panelists during online discussion forum in round 2 coupled with careful moderation of the forum by the moderators helped avoidance of an individual dominance that may result from strong verbalization or professional dominance, which may have led to biased results. This is often a concern in group-based approaches in qualitative studies, however anonymity and thoughtful direction helped prevent it in the study. In addition, anonymity allowed panelists to change their opinion on the basis of arguments presented by the other panel members during online discussion forum and avoided group pressure for conformity. These advantages of Delphi method are likely to increase reliability of consensus, as elucidated in previous studies. ${ }^{33,34}$

The study was limited by its small sample size and questions' generalizability. However, there is no gold standard of sample size for Delphi panels. Moreover, the sample size of this study aligns with the commonly observed number of experts involved in the previously published Delphi studies in hematology. ${ }^{35-38}$ In addition, the clinical scenarios were designed using typical practice patterns in the US and may not adequately reflect clinical variations encountered worldwide. Given that panelists volunteered and were compensated to participate in the study, self-selection bias may be present. However, study participants did not differ meaningfully in clinical expertise or practice setting from those who declined to participate. While we undertook a comprehensive literature search covering a full spectrum of challenging clinical 
scenarios, it is possible that relevant clinical scenarios were missed. Nevertheless, we anticipate that these study results provide unique views and valuable insights from the perspective of hematologists into current issues surrounding treatment decision-making for SPwHA.

\section{Conclusion}

The panelists achieved consensus for appropriate and inappropriate use of FVIII and emicizumab prophylaxis including, appropriate use of FVIII replacement therapy during and immediately post-surgery in addition to emicizumab prophylaxis for patients with elective surgery, and episodic FVIII followed by emicizumab prophylaxis for a newborn with postcircumcision bleeding. A majority of panelists preferred FVIII replacement therapy rather than emicizumab prophylaxis due to established evidence on safety, efficacy, and level of bleed protection for FVIII treatment. The recommendations emerging from this study may support or extend guidelines for practicing physicians when treating SPwHA without inhibitors. Further studies are needed to identify appropriate therapeutic approaches in those clinical cases for which consensus was not reached.

\section{Ethics Approval}

Using the Department of Health and Human Services regulations, 45 CFR 46.104(d)(2), Advarra Institutional Review Board (IRB) exempted this study from IRB oversight.

\section{Funding}

This study was funded by Takeda Pharmaceutical Company Limited, however, the sponsor had no role in the study, which involved design, execution of the Delphi panel, and analysis of study findings.

\section{Disclosure}

Sreenivas P. Veeranki was a former employee and Priti Pednekar, Marlon Graf, and Rifat Tuly are current employees of PRECISIONheor, a research consultancy to the health and life sciences industries. Sreenivas P. Veeranki is now affiliated with Optum LifeSciences, Eden Prairie, MN. Katharine Batt served as a consultant on this project through PRECISIONheor. Michael Recht's institutions have received research funding from Bayer, BioMarin, CSL Behring, Genentech, Grifols, Hema Biologics, LFB, Novo Nordisk, Octapharma, Pfizer, Sanofi, Spark, Takeda, and uniQure. He has served as a consultant to Catalyst Biosciences, CSL Behring, Genentech, Hema Biologics, Kedrion, Novo
Nordisk, Pfizer, Sanofi, Takeda, and uniQure. He serves on the Board of Directors for the Foundation for Women and Girls with Blood Disorders and Partners in Bleeding Disorders. The authors report no other conflicts of interest in this work.

\section{References}

1. Mahlangu J, Oldenburg J, Callaghan MU, et al. Health-related quality of life and health status in persons with haemophilia A with inhibitors: a prospective, multicentre, non-interventional study (NIS). Haemophilia. 2019;25(3):382-391. doi:10.1111/hae.13731

2. Chen SL. Economic costs of hemophilia and the impact of prophylactic treatment on patient management. Am J Manag Care. 2016;22 (5 Suppl):s126-s133.

3. Srivastava A, Brewer AK, Mauser-Bunschoten EP, et al. Guidelines for the management of hemophilia. Haemophilia. 2013;19(1):e1-e47.

4. Meeks SL, Batsuli G. Hemophilia and inhibitors: current treatment options and potential new therapeutic approaches. Hematology Am Soc Hematol Educ Program. 2016;2016(1):657-662. doi:10.1182/ asheducation-2016.1.657

5. Centers of Disease Control \& Prevention (CDC). Inhibitors and Hemophilia. Available from: https://www.cdc.gov/ncbddd/hemophi lia/inhibitors.html\#ref1. Accessed September 29, 2021.

6. Ayanian JZ, Landrum MB, Normand S-LT, Guadagnoli E, McNeil BJ. Rating the appropriateness of coronary angiography Do practicing physicians agree with an expert panel and with each other? N Engl J Med. 1998;338(26):1896-1904. doi:10.1056/ NEJM199806253382608

7. Campbell SM, Cantrill JA, Roberts D. Prescribing indicators for UK general practice: Delphi consultation study. BMJ. 2000;321 (7258):425. doi:10.1136/bmj.321.7258.425

8. Roberts M, Russell LB, Paltiel AD, et al. Conceptualizing a model: a report of the ISPOR-SMDM modeling good research practices task force-2. Value Health. 2012;15(6):804-811. doi:10.1016/j. jval.2012.06.016

9. Eubank BH, Mohtadi NG, Lafave MR, et al. Using the modified Delphi method to establish clinical consensus for the diagnosis and treatment of patients with rotator cuff pathology. BMC Med Res Methodol. 2016;16(1):56. doi:10.1186/s12874-016-0165-8

10. Armstrong C, Grant S, Kinnett K, et al. Participant experiences with a new online modified-Delphi approach for engaging patients and caregivers in developing clinical guidelines. Eur J Pers Cent Healthc. 2019;7(3):476-489.

11. Santaguida P, Dolovich L, Oliver D, et al. Protocol for a Delphi consensus exercise to identify a core set of criteria for selecting health related outcome measures (HROM) to be used in primary health care. BMC Fam Pract. 2018;19(1):152. doi:10.1186/s12875-018-0831-5

12. Santaguida PL, Oliver D, Gilsing A, et al. Delphi consensus on core criteria set selecting among health-related outcome measures (HROM) in primary health care. $J$ Clin Epidemiol. 2020;127:105-116. doi:10.1016/j.jclinepi.2020.04.028

13. Diamond IR, Grant RC, Feldman BM, et al. Defining consensus: a systematic review recommends methodologic criteria for reporting of Delphi studies. J Clin Epidemiol. 2014;67(4):401-409. doi:10.1016/j.jclinepi.2013.12.002

14. Boulkedid R, Abdoul H, Loustau M, Sibony O, Alberti C. Using and reporting the Delphi method for selecting healthcare quality indicators: a systematic review. PLoS One. 2011;6(6):e20476. doi:10.1371/ journal.pone.0020476

15. Birko S, Dove ES, Ozdemir V. Evaluation of nine consensus indices in Delphi foresight research and their dependency on Delphi survey characteristics: a simulation study and debate on Delphi design and interpretation. PLoS One. 2015;10(8):e0135162. doi:10.1371/journal. pone. 0135162 
16. National Hemophilia Foundation. MASAC Recommendations. Available from: https:/www.hemophilia.org/Researchers-HealthcareProviders/Medical-and-Scientific-Advisory-Council-MASAC /MASAC-Recommendations. Accessed July 2, 2020.

17. US Food and Drug Administration. Evaluating inclusion and exclusion criteria in clinical trials. FDA report; 2019.

18. Wood L, Bjarnason GA, Black PC, et al. Using the Delphi technique to improve clinical outcomes through the development of quality indicators in renal cell carcinoma. J Oncol Pract. 2013;9(5):e262e267. doi:10.1200/JOP.2012.000870

19. Meshkat B, Cowman S, Gethin G, et al. Using an e-Delphi technique in achieving consensus across disciplines for developing best practice in day surgery in Ireland. J Hosp Adm. 2014;3(4):1-8. doi:10.5430/ jha.v3n4p1

20. Colonna P, Andreotti F, Ageno W, Pengo V, Marchionni N. Clinical conundrums in antithrombotic therapy management: a Delphi Consensus panel. Int $J$ Cardiol. 2017;249:249-256. doi:10.1016/j. ijcard.2017.09.159

21. World Federation of Hemophilia. Guidelines for the Management of Hemophilia. 2020. doi:10.1111/hae.14046

22. Mannucci PM, Schutgens RE, Santagostino E, Mauser-Bunschoten EP. How I treat age-related morbidities in elderly persons with hemophilia. Blood. 2009;114(26):5256-5263. doi:10.1182/blood2009-07-215665

23. Coppola A, Tagliaferri A, Franchini M. The management of cardiovascular diseases in patients with hemophilia. Semin Thromb Hemost. 2010;36(1):91-102. doi:10.1055/s-0030-1248728

24. Dane KE, Lindsley JP, Streiff MB, Moliterno AR, Khalid MK, Shanbhag S. Successful use of emicizumab in a patient with refractory acquired hemophilia A and acute coronary syndrome requiring percutaneous coronary intervention. Res Pract Thromb Haemost. 2019;3(3):420-423. doi:10.1002/rth2.12201

25. Knoebl P, Sperr W, Schellongowski P, et al. Emicizumab for the treatment of acquired Hemophilia A: lessons learned from 4 very different cases. Hamostaseologie. 2019;39(S 01):SY13-SY15-AB.

26. Broderick CR, Herbert RD, Latimer J, et al. Association between physical activity and risk of bleeding in children with hemophilia. JAMA. 2012;308(14):1452-1459. doi:10.1001/jama.2012.12727

27. Zanon E, Tagliaferri A, Pasca S, et al. Physical activity improved by adherence to prophylaxis in an Italian population of children, adolescents and adults with severe haemophilia A: the SHAPE Study. Blood Transfus. 2020;18(2):152-158.
28. Mahlangu J, Oldenburg J, Paz-Priel I, et al. Emicizumab prophylaxis in patients who have Hemophilia A without inhibitors. $N$ Engl J Med. 2018;379(9):811-822. doi:10.1056/NEJMoa1803550

29. National Institute of Health. A clinical trial to evaluate prophylactic emicizumab versus no prophylaxis in Hemophilia A participants without inhibitors (HAVEN 3); 2016. Available from: https://clinical trials.gov/ct2/show/NCT02847637. Accessed September 29, 2021.

30. Roushan N, Meysamie A, Managhchi M, Esmaili J, Dormohammadi T. Bone mineral density in hemophilia patients. Indian $J$ Hematol Blood Transfus. 2014;30(4):351-355. doi:10.1007/s12288-013-0318-4

31. Sahin S, Sadri S, Baslar Z, Ar MC. Osteoporosis in patients with hemophilia: single-center results from a middle-income country. Clin Appl Thromb Hemost. 2019;25:1076029619861689. doi:10.1177/ 1076029619861689

32. Gamal Andrawes N, Hashem Fayek M, Salah El-Din N, Atef Mostafa R. Effect of low-dose factor VIII prophylaxis therapy on bone mineral density and $25(\mathrm{OH})$ vitamin $\mathrm{D}$ level in children with severe haemophilia A. Haemophilia. 2020;26(2):325-332. doi:10.1111/hae.13917

33. Gupta UG, Clarke RE. Theory and applications of the Delphi technique: a bibliography (1975-1994). Technol Forecast Soc Change. 1996;53(2):185-211.

34. Fletcher AJ, Marchildon GP. Using the Delphi method for qualitative, participatory action research in health leadership. Int J Qual Methods. 2014;13(1):1-18. doi:10.1177/160940691401300101

35. Bonnet P, Gringeri A, Gomperts E, et al. Modeling costs and outcomes associated with a treatment algorithm for problem bleeding episodes in patients with severe hemophilia A and high-titer inhibitors. Am Health Drug Benefits. 2011;4(4):219.

36. Martinoli C, Alberighi OD, Di Minno G, et al. Development and definition of a simplified scanning procedure and scoring method for Haemophilia Early Arthropathy Detection with Ultrasound (HEAD-US). Thromb Haemost. 2013;109(06):1170-1179. doi:10.1160/TH12-11-0874

37. Neufeld EJ, Solimeno L, Quon D, et al. Perioperative management of haemophilia B: a critical appraisal of the evidence and current practices. Haemophilia. 2017;23(6):821-831. doi:10.1111/hae.13279

38. Seuser A, Khayat CD, Negrier C, Sabbour A, Heijnen L. Evaluation of early musculoskeletal disease in patients with haemophilia: results from an expert consensus. Blood Coagul Fibrinolysis. 2018;29 (6):509. doi:10.1097/MBC.0000000000000767
Journal of Blood Medicine

\section{Publish your work in this journal}

The Journal of Blood Medicine is an international, peer-reviewed, open access, online journal publishing laboratory, experimental and clinical aspects of all aspect pertaining to blood based medicine including but not limited to: Transfusion Medicine; Blood collection, Donor issues, Transmittable diseases, and Blood banking logistics; Immunohematology; Artificial and alternative blood based therapeutics; Hematology; Biotechnology/nanotechnology of blood related medicine; Legal aspects of blood medicine; Historical perspectives. The manuscript management system is completely online and includes a very quick and fair peer-review system. Visit http://www.dovepress.com/testimonials.php to read real quotes from published authors. 